point or the other assumptions that underlie the statement that the method I have outlined gives the true systolic pressure in the radial artery. I do, however, wish to point out that all that can be asserted is that the systolic pressure was $120 \mathrm{~mm}$. in the artery observed at the moment of observation.

From the fact that in healthy young adults in the recumbent posture the systolic pressure in the posterior tibial artery is fonnd to be about the same as in the radial when similarly measured, there seems to have arisen a supposition, tacit perhaps rather than avowed, that the systolic pressure in all the arteries of the body would be much the same. This is a very large supposition, and, as I believe, an erroneous one.

Having already demonstrated that a record of systolic pressure is of slight value in the absence of a simultaneous record of diastolic pressure, we next have to ask, What are the instruments which afford the information that we scek? Instruments recording both pressures are few in number, and of these the best are the sphygmomanometer of Erlanger and the sphygmo-oscillometer of Pachon. For graphic records of physiological blood pressure in suitable cases Erlanger's apparatus has considerable utility. In clinical medicine, nevertheless, its employment is contraindicated, since it is too complicated, cumbersome, and expensive for purposes other than those of the laboratory.

No such objections apply to Pachon's sphygmo-oscillometer, which is less expensive, easily portable, and simple in application. Moreover, if properly used, it eliminates the personal equation, so that, having now used this instrument for upwards of three years, and having tested it against a large number of other apparatus, $I$ am able to say that with it I have obtained very useful clinical rosults. By this I do not mean to say that I consider it a perfect instrument, but it is certainly quite a good one, and both in private work and in hospital I use it in preference to any other. One minute only is required for an observation, which, however, I always repeat for the sake of accuracy, and at the end of this short time one hes a complete record of maximal pressure, minimal pressure, and amplitude of pulse wave.

In stating that the Pachon sphygmo-oscillometer gives a record of maximal and minimal pressures, I do not say that even these figures represent the real blood pressures of the case under observation. More investigation on this point is required than I have yet been able to make. The salient fact is that both pressures are obtained simul. taneously by the use of the same method, and that these are relative one to the other, thus affording exceedingly valuable clinical comparisons over large numbers of cases. It is of no avail to measure one pressure by a mercurial manometer, for example, and another by the method of oscillations, for readings of the systolic pressure by one method and of the diastolic by another method totally different in principle fail to give results which are sufficiently accurate to be comparable with advantage. In fine, my opinion is that an absolutely satisfactory means of recording at the bedside the various blood pressure data has yet to be discovered.

To a future occasion I must leave the remarks which I had hoped to make on readings taken in the manner I have described as guides to prognosis and treatment. It has been denied that blood pressure records can give any useful indications in these respects; but, again, this pronouncement has been made from consideration of systolic apart from diastolic pressures. Personally, I may say that $I$ often find readings taken at frequent intervals of considerable assistance when considered in conjunction with the other available physical signs and symptoms. For instance, if in a case of arterio-sclerosis both pressures are rising in spite of treatment, the import of this is unfarourable. In a patient of mine, a lady aged 65 , the subject of hypertrophy of the left ventricle, generalized arterio-sclerosis, and some chronic interstitial nephritis, the arterial blood pressures, systolic and diastolic, have been throughout the past two years very high. Some nine months ago the pressures rose, and I considered her to be in danger of cerebral apoplexy. This event speedily happened, being associated with paresis of face, arm, and leg on the same side, from which now the patient has made very fair rccovery, and the blood pressure has become slightly lower than the original figures. As regards treatment, the case of a schoolboy, aged 14, with well-marked double aortic disease, is of interest. On lis first visit the readings were $20-2-8$, the first being the systolic pressure in centimetres $\mathrm{Hg}$, the last the diastolic, and the intermediate figure the amplitude of pulse ware. Three weeks later the readings were $14-0.5-7$, the general condition and state of the circulatory system having correspondingly improved.

It would be easy to multiply examples of general diseases as well as of circulatory affections, but, since I liope to deal with these in a later paper, I will conclude with the recommendation that, as opportunities present themselves, you will test for yourselves the importance of registering diastolic as well as systolic pressures.

REFERENCES.

1Halls Dally: Pachon's Sphygmo-oscillometer and its Use in the Determination of Blood l'ressure, Lancet, September 2nd, 1911, Janewas: The Clinical Study of Blood Pressure. New York and

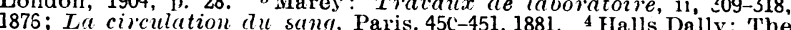
Clinical Determination of Conot ${ }_{5}$ Russell Wells and Leonard Hill: Proc , October 7th, 1911, p. 813. 6 Russell, William: The Clinieal Estimation of , rol. Ixxxvi, 1913. BRITISH MIDICAL JOCIRAL, October 10th, 1908, p. 1076.

\section{THE IDIO-VENTRICULAR RHYTHM.}

$$
\text { A CLIAICAL CASE WITI POLYGRAPHC }
$$

RECORDS.

Br CHARLES ALLEN ROBINSON, B.A., M.B., B.C.CANTAB.

LEOMIXSTER, HEREFORDSHIRE.

THe term "bradycardia" is applied correctly to those cases of infrequent heart action in which the auricles participate in the infrequency. It may be either normal or of the so-called nodal type.

In the normal bradycardia the auricular contractions bear the normal time relationship to those of the ventricular. The term " nodal bradycardia" is applied to that form in which the auricular contractions occur simultaneously with, or very slightly before or after the ventricular contractions. The case on which this paper is based may be regarded as one primarily of bradycardia, and it has some features in common with nodal brady. cardia as defined. It differs, however, in this, that the polygraphic records show a regularly-recurring auricular contraction to which the ventricle fails to respond.

In cases in which the conduction of impulses is not interfered with, the idio-ventricular whythm asserts itself, provided the period of time occupied by the rhythmic formation of impulses is less in the ventricles than in tho auricles. So that a lengthening of the time of stimulus formation at the sinus, or a lessening of the time in the ventricles, or a combination of these, will produce the assumption of the idio-ventricular rhythm.

In the case of bradycardia we have, then, an essential element for the production of the idio-ventricular rhythm, provided the bradycardia is sufficiently pronounced, and the period of time occupied by stimulus formation in the ventricle is not also lengthened sufficiently to prevent the ventricular rhythm from coming into action.

A Casc Iilustrating Idio-rentricular Rhythm.

On December 5th, 1912, a patient came to me complaining that he was unable to continue his work because of pains in the chest, arms, anc legs, and some breathlessness. He is a blacksmith by trade, aged 32 years. He has been at this trade for the last ten vears, but previously to that from the age of 16 years had been emplored in a coal mine. He is a small man and not of good muscular development.

There is no history of illness or injury likely to cause affection of the heart. He denies having had syphilis. He has had no attacks of fainting.

He has a troublesome cough, with some expectoration.

The pulse is small and at the rate of 40 per minute, and is almost regular in rhythm. There is no evidence of atheroma. The heart sounds are clear but faint, and they correspond to the beats of the radial pulse.

The lungs are emphysematous, which prevents the size of the heart being determined. There are no signs in the lungs to account for the cough. There is some tenderness and increased muscular resistance in the region of the liver.

There is no oedema of the extremities or other signs of heart failure other than those mentioned.

The urine does not contain albumin. 
On December 5th, 10th, and 16th tiracings similar to that shown in the figure were taken. In the venous curve of the figure the $a$ and $c$ waves occur almost simultaneously, resulting in a rise with two pealis. Measurement shows that the first of the two pealss is due to systole of the ventricle, and it is probable that the second is due to auricular contraction, though it must not be lost sight of that it may be due to instrumental error. The whole wave is, however, too broad to be entirely due to ventricular contraction. The combined wave causes a large excursion of the recording pen, especially when compared with the radial pulse wave. This is usual in cases when the $a$ and $c$ waves fall together. It was

grade stimulation, and is not due to sinus stimulation, and the reason for this is the constant time relation between $a$ and $c$. If $a$ and $c$ were due to stimuli arising quite independently-namely, $c$ from an idio-ventricular stimulus fornation and $a$ from a stimulus arising at the sinus-it is not likely that they would bear such a constant time relationship to one another as they do, over a period of days at least.

If, then, $a$ in $c a$ is not due to sinus stimulation, it is unlikely that $a$ wili be either, and for a like reasonnamely, that if it arose from a cause entirely separated from that of $a$ in $c a$, it would not be likely to bear such a constant time relation to it over so long a time. On the other hand, if it is due to an extrasystole of the auricle, its constant time relation to $a$ in $c a$ is reasonable. The possibility of the wave $a^{\prime}$ being due to variations of r to passive movements of the

difficult to obtain a record of the radial pulse wave at all, as the wave was so feeble. These larger elevations due to the $a$ and $c$ waves follow at regular intervals, and are succeeded by smaller waves, $v$, which measurement shows are due to filling of the auricle, the fall of the wave being coincident in time with the opening of the auriculoventricular valves. Following the $v$ waves is in many instances a sharp though small rise in the tracing, followed by a very small fall. This small wave is most likely d ue to the closing of the auriculo-ventricular valves, due to the inrush of blood into the ventricle (the $b$ wave of Gibson). After this wave comes another (marked $a^{\prime}$ ) not very pronounced, but which is nevertheless distinct and frequently present.

A great deal of the interest of these tracings centres upon the interpretation of this wave. It recurs with sufficient constancy and distinctness to render it unlikely that it is due to instrumental error or manipulation.

It occurs at a point on the tracing subsequent to the time when the pressure in the veins having risen on account of the auricle filling, giving rise to the wave marked $v$, and the pressure having fallen due to the opening of the auriculo-ventricular valves in consequence of the ventricular diastole having begun, the pressure in the auricles again rises on account of the continued inflow of blood into them. It occurs, in fact, when the ventricle is in diastole, and is filling with blood. This raises the presumption that it is due to auricular contraction.

If it were due to the continued passive filling of the ventricle, auricle, and veins, the rise in the wave might be expected to continue to occur until the occurrence of the sudden rise of the curve due to the following ventricular contraction. There is, however, a very definite fall of pressure, as indicated by the descent of the wave, which seems to indicate the relaxation of the auricle after its contraction.

It bears a very constant relation to the preceding ventricular and auricular contractions; moreover, the period from $a$ to $a$ ! is about three-fifths of a second, and that from $a^{\prime}$ to the succeeding $c$ is about four-fifths of a second. The former period is very constant; the latter varies somewhat. Now, whether $a$ (in the wave $c a$ ) is due to an auricular contraction from a normally produced stimulus or to a retrograde stimulus from ventricular contraction causing an auricular contraction, which at the same time abolishes the normally produced stimulus material at the sinus, $a^{\prime}$ should follow it after a period a little longer than from $a^{\prime}$ to $c$, because $c$ occurs before the normally produced stimulus can produce the auricular contraction causing $a$, unless there is a sinus arrhythmia of alternately long and short periods of a very regular character-that is to say, an auricular bigeminy-the second auricular contraction of the bigeminy not being followed by a ventricular contraction. Another explanation of the bigeminy is that the wave $a^{\prime}$ is due to a heterogenetic auricular contraction or extrasystole; its constant time relationship to the preceding contractions of ventricle and auricle being due to there being some causal connexion between them. The question, then, arises, Is the bigeminy of the auricle due to a sinus arrhythmia, or is it due to auricular extrasystole? The auricular contraction producing $a$ in $a c$ is due to retro. ventricle and auriculo-ventricular valves caused by the onflow of blocd from the veins, cannot be altogether dismissed, the length of time occupied by ventricular diastole accounting for the fact that the waves due to the variations of pressure are so noticeable. If this is so, the sequence of events is as follows: At $v$ the pressure falls due to the opening of the auriculo-ventricular valves, after which there is a sudden rise in pressure in the ventricle causing a closure of the valves producing the short wave occasionally distinctly seen just before $a^{\prime} ; a^{\prime}$ is produced by the continuance of the filling of the auricle, and the fall represented by its descending limb is due to a second opening of the valves. On the whole, I think it must be admitted that the wave $a^{\prime}$ is not due to variations of pressure due to passivo movements of the ventricle and auriculo-ventricular valves. The pressure needed to open the valves under these conditions must be very slight, and could not be sufficient to cause a ware such as $a^{\prime}$ represents in the venous pulse. The assumption that it is due to an auricular contraction offers an explanation of the constant time relationship which the wave $a^{\prime}$ bears to the previous c a wave, if tho mechanism of a bigeminal action due to premature or extrasystolic contraction of the auricle bo accepted.

The outstanding feature of the polygraphic tracing is the infrequency of the radial pulse.

What is the explanation of this infrequent pulse-rate? The commonest cause of a pulse beating at the rate of 40 to the minute, or thereabouts, is the occurrence of an extrasystole succeeding each normal beat, the ventricular contractions of the extrasystole being too feeble to send the pulse-wave to the wrist, or that the extrasystole arises in the auricle and the passage of the impulse to the ventricle is blocked.

Another explanation to be offered is that the case is one of heart-block, either complete, or in which every other impulse is blocked at the auriculo-ventricular junction. It may be advanced as an explanation that a stimulus normally produced, and giving rise to the wave at $a^{\prime}$, is so long delayed by impaired conduction that the ventricular contraction resulting from it does not take place until a very short interval of time before the succeeding auricular contraction. If this is so the $a-c$ interval is an extra ordinarily long one, namely, about $\frac{3}{5}$ to $\frac{4}{5}$ of a second. The normal $a-c$ interval is from $\frac{1}{1 \cdot \sigma}$ to $\frac{2}{10}$, and pathologically the longest recorded is about 5 (Lewis). Complete heart-block is unlikely, though by no means im. possible, with a pulse frequency of 40 to the minute. It is also unlikely on account of the general condition of the patient, and the fact that there have been no fainting attacks. To suppose that it is due to complete heartblock is to assume that, although the auricle and ventricle are beating independently, the ventricular contraction happens to fall every time at an alternate auricular contraction, which is to assume the occurrence of a too great coincidence. That the case is not one of true bigeminy, as defined by Professor Wenckebach, is obvious from the fact that the effective contraction does not originate at the sinus.

There may, however, be a bigeminal contraction of the auricle which is not followed by a ventricular systole. 
The case may be classed as one of nodal bradycardia, being one of those uncommon ones in which the pulse beat is regular. If we assume that the ware $a^{\prime}$ is due to auricular contraction, and in the light of that interpretation we look for an explanation of the tracings taken on December, 5th, 10th, and 16th, we see that a wave due to auricular systole, whether resulting from an impulse normally produced at the region of the sinus or in the nature of a bigeminy due to auricular extrasystole, appears in a position intermediate between those due to the ventricular contractions. But because conduc tion is impaired his fails to produce a ventricular contraction.

The failure of the ventricle to contract can be explained as clue partly to the refractor'y state of the ventricle from its previous contraction, the period between the end of the ventricular systole which has occurred and the beginning of the next $a^{\prime}$ wave being $1 \frac{1}{2}$ fifths only. 'The ventricle is, however, at the time of the arrival of the impulse from the auricle in a state of diastole, and should therefore be in a condition capable of contraction. The fact that it does not occur, therefore, shows some degrec of impairment of conduction or excitability of the musculature. 'The difficulty arises that $a^{\prime}$ is not followed by a wave due to ventricular systole on account of block. but the ventricular systole is able to produce an amicular contraction by retrograde stimulation.

At the same time it is difficult to conceive that the ventricular systole should always bear the same time relationship to auricular systole that it does, over so long a time, unless there is some direct connexion between them. What the connexion is, unless it is retrograde stimulation from ventricle to auricle, it is difficult to say. If there were such pathological changes in the junctional tissues that no stimulus could pass from auricle to ventricle, there would be complete dissociation of auricular and ventricular rhythms, and they would not bear a constant time relation to one another.

Although, however, the power of conduction is so far reduced by organic changes, or by nerve influence, as to prevent $a^{\prime}$ being followed by ventricular systole, on account of the proximity of the previous ventricular contraction, by the time the next ventricular systole occurs it may have sufficiently recovered to permit a retrograde stimulus to cause an auricular systole.

However this may be, this contraction of the auricle, not having been responded to, a spontaneous contraction of the ventricle takes place before a normally produced auricular contraction is due. As a result the wave due to the ventricular occurs before that due to the auricular contraction.

During the period following the previous ventricular contraction stimulus material has been formed in it, and the ventricular rinscle has been recovering from its refractive state, the two conditions approaching a state suitablo for producing a homogenetic idio-ventricular contraction. The time taken for the process to be completed normally in the ventricle is at least $7 \frac{1}{2}$ fifths of a second. In the present case the period between the end of the ventricular contraction and the beginning of the next is about $5 \frac{1}{2}$ fifths. The explanation of the fact that the sinus rhythm controls the ventricular in the normal mechanism is that the formation of stimulus material at the sinus takes place at a greater rate in it than it does elsewhere in the heart. If for any reason the idioventricular rhythm is not dormant, and the periods of time between the formation of stimuli in it are shorter than in the sinus, the ventricular rhythm will predominate.

In the present case the idio-ventricular rhythm is not dormant, and the rate of stimulus formation in the ventricle being more rapid than is usual, combined with the fact that no effective stimulus has reached the ventricle for so long a time, the ventricle escapes every cardiac cycle, as seen on the tracings of December 5th, 10th, and 16 th.

This ventricular systole due to the escape of the ventricle causes a retrograde stimulation of the auricle to contract. The conclusion is irresistible that it is no mere chance or coincidence that the ventricular contraction shoulc talke place at about the time of the normally-produced stimulus of the auricle with such great regularity.
For this reason I conclude that a retrograde stimnlation of the auricle has occurred. In this way the regularity of the rhythm is maintained and the condition is produced of the ventricular contraction, which is effectivo in maintaining the circulation, arising in an abnormal situation.

Tracings taken on several occasions subsequently have shown a perfectly regular pulse, though somewhat infrequent, and in all other respects normal both in regard to the venous and radial curves.

It follows, therefore, that if the degree of heart-block which resulted in the second of the bigeminal auricular contractions not being followed by a ventricular reponse was due to organic lesions in the tissues of the heart responsible for the conduction of impulses, those lesions have been removed.

Cases of heart-block have been recorded in which the block has been due to a haemorrhage from traumatism or. spontaneous rupture of an atheromatous artery in tho neighbourhood of the auriculo-ventricular bundle. It is conceivable that such a lesion conld clear up sufficiently to allow the subsequent passage of impulses. The case discussed may have beeri due to haemorrlage in the bundle caused by such a condition. But this is unlikely in the absence of any listory of injury, and in consideration of the patient's age and the absence of evidence of atheromatous arteries elsewhere.

The conclusions arrived at from a study of the polygroplic tracings are that the infrequency of the pulse-rate is due primarily to a relative delay in the rate of stimulus formation at the sinus. This, together with an extrasystolic contraction of the auricle not followed by a ventricular response, causes the period before effectire stimulation of the ventricle coming from the normal situation to be so long that the ventricle escapes, and in turn produces auricular contraction by a retrograde stimulation, thus producing the establishment of the idio-ventricular rhythm.

\section{FATAL PURPURA FOLLOWING SCARIET FEVER. \\ $B Y$}

J. BIERNAC'KI. M.D.,

PHYSICIAN SUPERINTENDENT, platstow FEVER hospital, LONDON, E. AND

A. L. DYKES, M.D.,

SENIOR ASSISTANL MEDICAL OFFICER, PIAASTON FITLER HOSPITAL, LONDON, $\mathrm{E}$

Relatively rarer than the mild purpura chiefly affecting the lower extremities which occurs after scarlet fever is a malignant form with extensive superficial lesions in various parts of the body. The case here reported belongs to the latter type, but is unique in the writers' experience in that the purpuric areas showed a definite inflammatory element. The inflammation was revealed not only by an undue degree of tenderness, but by marked sivelling of the loose tissues, and a certain amount of induration. Early in the attack it seemed likely that the lesions would become gangrenous, but the patient died before any breaking down had occurred; also it was noticed before death that at points on the right leg and left arm the purpura was beginning to retrogress.

The patient was a boy aged 6 years, who was admitter to Plaistow Hospital with scarlet fever. There was a history of measles, chicken-pox, and whooping-cough within the two previous years; as a result, it was stated that he became anaemic, but that otherwise his health was good. There was nothing noteworthy in his family history.

At the time of aclmission the attack of scarlet fever was declining. The case ran the ustal course, without complications, and by the end of the fifth week the patient was up, and seemed in ordinary health save for the persisting anaemia. Iron and cod-liver oil were prescribed. In the seventh week moderate tonsillitis set in. The boy's temperature rose to $100^{\circ} \mathrm{F}$. He was liept in bed, and in two days the pyrexia disappeared. The tonsillitis, streptococcic in origin, also rapidly subsided. The patient, how. ever, was not allowed up as the anaemia liad slightly 